\title{
Additional Content
}

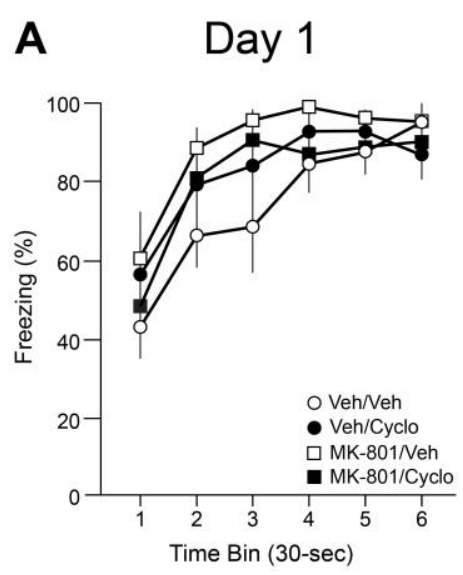

D
B

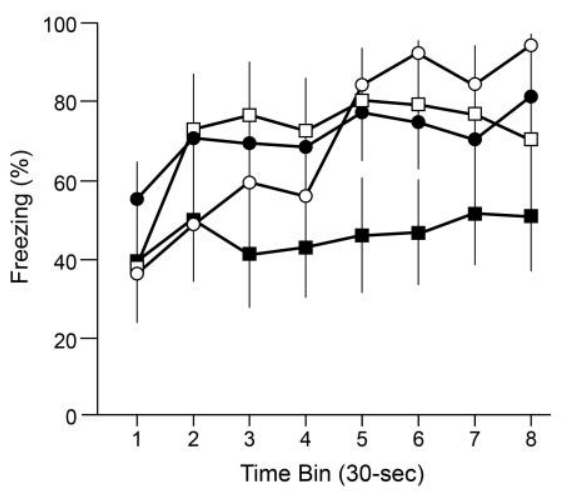

Day 4

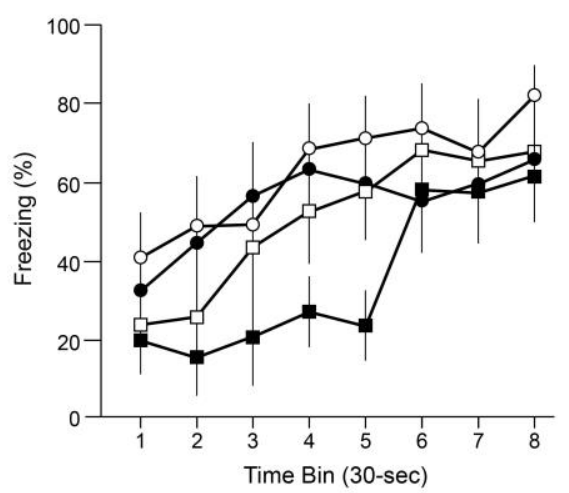

Day 2

E
C Day 3

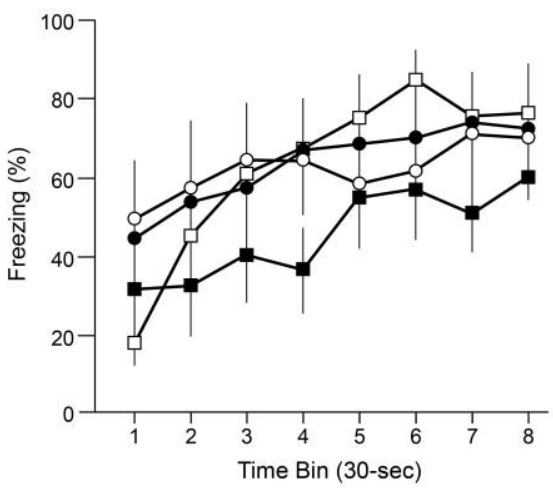

Day 5

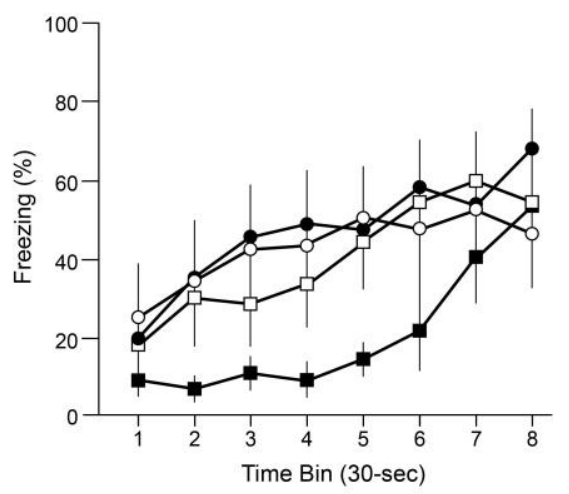

Figure S1. The number of animals in each group was as follows: vehicle/vehicle $=6$, vehicle/cycloheximide $=10$, MK-801/vehicle $=9$, MK-801/cycloheximide $=8$. Average percent time spent freezing for each 30 -second period of each extinction session. Bars depict \pm 1 SEM. Panel A depicts a three-minute test originally intended to be a standalone test session with shorter duration (3 minutes instead of 4 minutes). All subsequent extinction sessions had a duration of four minutes. (A) On day 1 , there was a main effect of extinction bin $[F(5,145)=29.07, p<0.001]$, but no other main effects or interactions [all $p s>0.05$ ]. (B) On day 2, there was a main effect of extinction bin $[F(7,203)=7.15, p<0.001]$ and an infusion $x$ extinction bin interaction, $[F(7,203)=2.13$, $p<0.05$ ]. There were no other main effects or interactions [all $p s>0.05$ ]. (C) On day 3 , there was a main effect of extinction bin $[F(7,203)=11.82, p<0.001]$, but no other main effects or interactions [all $p s>0.05$ ]. (D) On day 4 , there was a main effect of extinction bin $[F(7,203)=12.54, p<0.001]$, but no other main effects or interactions [all $p s>0.05]$. (E) On day 5, there was a main effect of extinction bin $[F(7,203)=11.86, p<$ 0.001 ], but no other main effects or interactions [all $p s>0.05$ ]. 
A

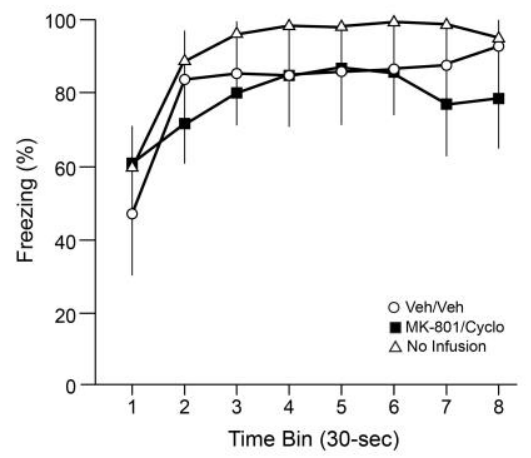

D

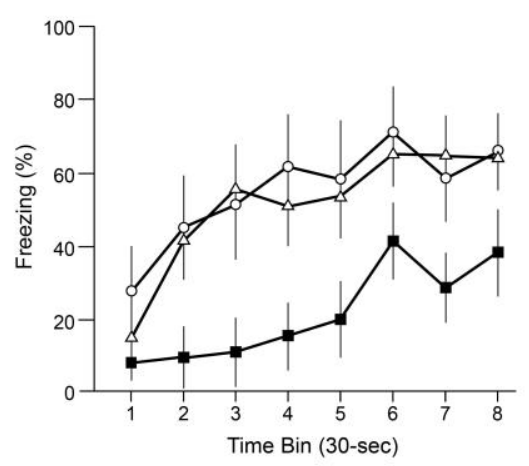

B

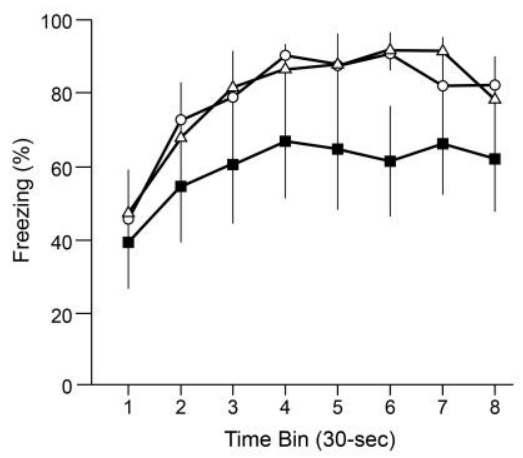

E

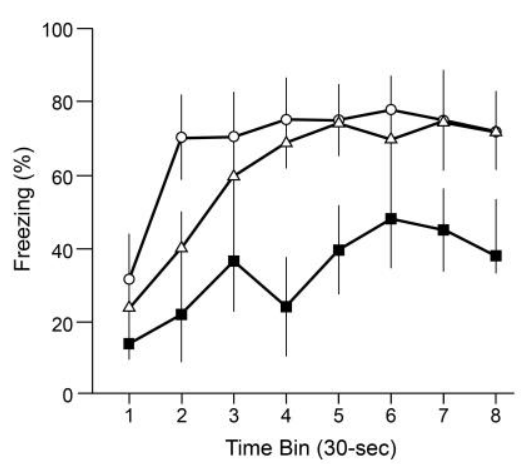

C

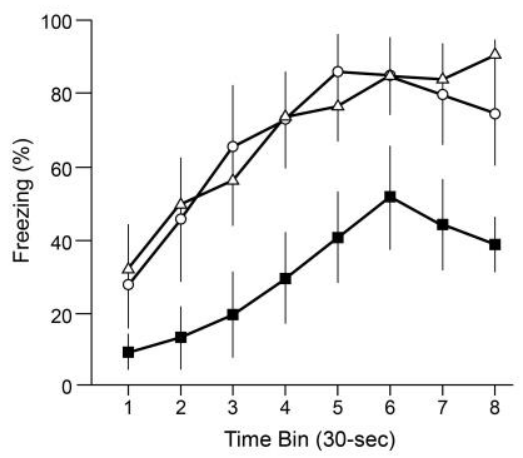

$\mathbf{F}$

Day 6

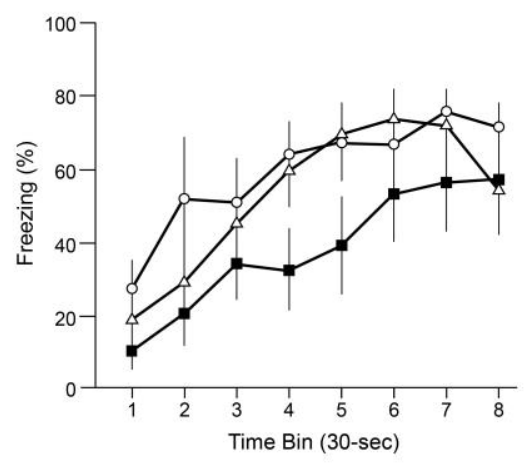

Figure S2. The number of animals in each group was as follows: vehicle/vehicle $=7$, MK-801/cycloheximide $=8$, no surgery $=10$. Average percent time spent freezing for each 30-second period of each four-minute extinction session. Bars depict \pm 1 SEM. (A) On day 1 , there was a main effect of extinction bin $[F(7,154)=13.93, p<0.001]$, but no other main effects or interactions [all $p s>0.05$ ]. (B) On day 2, there was a main effect of extinction bin $[F(7,154)=9.82, p<0.001]$, but no other main effects or interactions [all $p s>0.05$ ]. (C) On day 3 , there was a main effect of extinction bin $[F(7,154)=19.67, p<0.001]$ and a main effect of condition $[F(2,22)=5.63, p<0.05]$. There were no other main effects or interactions [all $p s>0.05$ ]. (D) On day 4, there was a main effect of extinction bin $[F(7,154)=9.04, p<0.001]$ and a main effect of condition $[\mathrm{F}(2,22)=5.17, p<0.05]$. There were no other main effects or interactions [all $p \mathrm{~s}>$ $0.05]$. (E) On day 5 , there was a main effect of extinction bin $[F(7,154)=10.93, p<$ $0.001]$ and a main effect of condition $[F(2,22)=5.07, p<0.05]$. There were no other main effects or interactions [all $p s>0.05]$. $(F)$ On day 6 , there was a main effect of extinction bin $[F(7,154)=17.84, p<0.001]$, but no other main effects or interactions [all ps > 0.05]. 